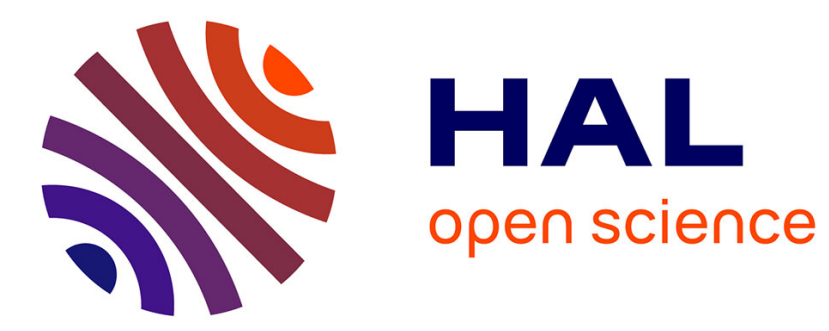

\title{
On-the-Fly Analysis of Distributed Computations
}

Eddy Fromentin, Claude Jard, Guy-Vincent Jourdan, Michel Raynal

\section{To cite this version:}

Eddy Fromentin, Claude Jard, Guy-Vincent Jourdan, Michel Raynal. On-the-Fly Analysis of Distributed Computations. [Research Report] RR-2595, INRIA. 1995. inria-00074088

\section{HAL Id: inria-00074088 \\ https://hal.inria.fr/inria-00074088}

Submitted on 24 May 2006

HAL is a multi-disciplinary open access archive for the deposit and dissemination of scientific research documents, whether they are published or not. The documents may come from teaching and research institutions in France or abroad, or from public or private research centers.
L'archive ouverte pluridisciplinaire HAL, est destinée au dépôt et à la diffusion de documents scientifiques de niveau recherche, publiés ou non, émanant des établissements d'enseignement et de recherche français ou étrangers, des laboratoires publics ou privés. 


\section{On-the-fly analysis of distributed computations}

Eddy FROMENTIN, Claude JARD,

Guy-Vincent JOURDAN, Michel RAYNAL

$\mathbf{N}^{\circ} 2595$

Juin 1995

PROGRAMME 1 



\title{
RINRIA
}

\section{On-the-fly analysis of distributed computations*}

\author{
Eddy FROMENTIN, Claude JARD, \\ Guy-Vincent JOURDAN, Michel RAYNAL \\ Programme 1 - Architectures parallèles, bases de données, réseaux \\ et systèmes distribués \\ Projets Adp et Pampa
}

Rapport de recherche $\mathrm{n}^{\circ} 2595$ - Juin 1995 - 21 pages

\begin{abstract}
At some abstraction level a distributed computation can be modeled as a partial order on a set of observable events. This paper presents an analysis technique which can be superimposed on distributed computations to analyze the structure of control flows terminating at observable events. A general algorithm working on the longest control flows of distributed computations is introduced. Moreover it is shown how this algorithm can be simplified according to the distribution of observable events or to the kind of property we want to recognize.
\end{abstract}

Key-words: Distributed computation, observable event, control flows, causal precedence, sequence analysis.

(Résumé : tsvp)

*This paper appeared in Information Processing Letters, 54, pp. 267-274, May 1995 


\section{L'analyse au vol des exécutions réparties}

Résumé : Un calcul réparti, à un certain niveau d'abstraction, peut être modélisé par un ordre partiel sur un ensemble d'événements observables. Cet article présente une technique d'analyse qui peut être superposée au calcul réparti, afin d'analyser la structure du flot de contrôle aboutissant à chaque événement observable. On introduit un algorithme général travaillant sur les plus long flots de contrôle. On montre de plus que cet algorithme peut se simplifier suivant la répartition des événements observables ou le type de motif que l'on veut reconnaître.

Mots-clé : Exécution d'un programme réparti, événements observables, flot de contrôle, précédence causale, analyse de séquences. 


\section{Contents}

1 Introduction 3

2 Model of distributed computations $\quad \mathbf{5}$

2.1 Causal precedence............... 5

2.2 Abstraction level and observable events . . . . . . . . . . 6

3 Specification of properties $\quad \mathbf{8}$

3.1 Language associated with observable events . . . . . . . . 8

3.2 Specifying properties . . . . . . . . . . . . 10

4 An on-the-fly analysis algorithm 11

4.1 An incremental analysis . . . . . . . . . . . . . 12

4.2 Principle of the algorithm . . . . . . . . . . . . . . 13

4.3 Construction of the algorithm . . . . . . . . . . 13

5 Particular Cases $\quad \mathbf{1 8}$

5.1 Non invisible process participation . . . . . . . . . . . 18

5.2 Non invisible communication . . . . . . . . . . . . . . 18

6 Concluding remarks $\quad 19$

\section{Introduction}

One important topic addressed by computer science is the analysis of sequences of symbols or words (e.g. syntactical analysis, pattern recognition, etc). Wellsuited formalizations have been designed and specialized tools have been implemented to make feasible such analyses in specific domains (formal languages and automata theory are the most famous example of these works).

Computations performed by distributed computing systems do not yield a linear sequence of events. The relationship between events inherently define a partial ordering. For a particular computation, events produced by each process are totally ordered and communications creates dependencies among events belonging to distinct processes. Since Lamport's seminal paper [8], this partial order relation on events is generally called happened before (with respect

$\operatorname{RR} n^{\circ} 2595$ 
to a logical time frame) or causal precedence. It formally expresses control flows and their mutual dependencies which organize the distributed execution.

In this paper we are interested in analyzing "on-the-fly" the set of "words" produced by a distributed computation; a word being defined from sequences of observable events produced by an execution of a distributed program. The practical motivation of our work comes from debugging, testing and monitoring of distributed systems [5, 7]. In this context we choose to explore analysis techniques which must be run on-the-fly and without delay (this is particularly important in the context of reactive monitoring). These constraints eliminate the possibility to log events produced by each process (as the analysis cannot be done off-line) or to use an additional process (monitor) that would receive notification messages sent by processes of the computation in order to analyze their traces (in that case notification messages would add some delay between event occurrences and their knowledge by the monitor). In other words we constraint our analysis mechanism to be superimposed on the computation and to use only a piggybacking technique to convey analysis related information from one process to another.

According to the aim of the analysis (detection of a property, for example) only a subset of all the events generated by a distributed execution are meaningful to the user: these events are called "observable events". From this point of view, the other events are ignored at the abstraction level considered; they participate only in the establishment of causal dependencies between observable events. Sequences of observable events are defined by the chains of the partial order relation associated with the computation. Although each observable event of the computation is unique, several events can be occurrences of the same action. So a labeling function is introduced and the sequences of observable events are associated with words (concatenation of labels). The properties considered in this paper are described as finite state automata. The analysis consists in checking whether the pattern of actions defined by the automaton occurs in the words defined by the considered computation. The analysis is carried out on-the-fly without delay on these words. Other kinds of automata could be used but finite state automata are sufficient to illustrate our analysis technique and to solve efficiently practical problems.

The paper is divided into four main sections. Section 2 presents our model of distributed computations. Section 3 presents the kind of specification 
or properties we are interested in, and gives their formal interpretation: the definition of languages (set of words) associated with distributed computations and the basic question (satisfaction rule) which can be answered by the analysis. Section 4 presents a general distributed algorithm which, in this context, analyzes on the fly and without delay a distributed computation. Section 5 examines particular cases according to the position of observable events with respect to communication events.

\section{Model of distributed computations}

\subsection{Causal precedence}

Distributed computations result from the execution of distributed programs. A distributed program is made of $n$ sequential processes $P_{1}, . ., P_{n}$ which synchronize and communicate by the only mean of message passing. A distributed program can be directly written by a programmer or can be the result of the compilation of a parallel or sequential program for a distributed memory parallel machine. Processes that realize the distributed computation execute actions which are either communication actions (sending of a message, reception of a message) or internal actions (all the other actions). Each execution of an action constitutes an event.

The activity of a process $P_{i}$ is perceived as a set of local atomic events $E_{i}$, totally ordered by a local precedence relation $<_{i}$. This set $E_{i}$ can be partitioned into two subsets:

- $I_{i}$ : the set of internal events of $P_{i}$ (resulting from internal actions);

- $X_{i}$ : the set of communication events of $P_{i}$ (send and receive events).

The set $E=\bigcup_{i} E_{i}$ of all the events produced by the distributed execution is partially ordered by Lamport's relation called happened before or causal precedence [8], denoted by $\leq_{E}$.

RR $\mathrm{n}^{\circ} 2595$ 


$$
\begin{gathered}
\forall x \in E_{i}, y \in E_{j}: x \leq_{E} y \stackrel{\text { def }}{=} \\
\begin{cases}\text { or } & x=y \\
\text { or } & i=j \text { and } x<_{i} y \\
\text { or } & x \text { is the sending of a message and } y \text { its reception } \\
& \exists z \text { such that } x \leq_{E} z \text { and } z \leq_{E} y\end{cases}
\end{gathered}
$$

\subsection{Abstraction level and observable events}

Analyzing a (distributed) computation requires to define precisely the abstraction level we consider (usually language, system or hardware level). In our approach, this level is defined by the set of events that must be observed in order to check the property defined by the user. So we consider here that, for a given abstraction level, only a subset of internal events are relevant, and result from execution of specific actions (for example, modifications of some process variables). These events are called observable. Communication events create causal dependencies between observable events but are supposed to be not observable. This observation notion defines a screen that filters out all irrelevant events while keeping all causal dependencies between relevant events.

Let $O_{i} \subseteq I_{i}$ be the set of observable events of $P_{i}$ and $O=\bigcup_{i \in 1 . . n} O_{i}$. At the considered abstraction level, the distributed computation is characterized by the poset $\left(O, \leq_{0}\right)$ with $\leq_{0}$ defined by ${ }^{1}$ :

$$
\forall x, y \in O: x \leq_{O} y \Leftrightarrow x \leq_{E} y
$$

Distincts events can be executions of a same action. So each event is labeled by the name (label) of the corresponding action. These labels constitute the atoms of the language used to specify properties. Let us denote $\Sigma$ the finite set of labels and $\lambda$ the labeling function from $O$ to $\Sigma$.

In the sequel, posets $\left(O, \leq_{0}\right)$, labeled with $\lambda$, are represented by sets of words of $\Sigma^{\star}$. As there is no satisfactory method for coding a labeled poset as a set of strings when vertices with the same label are not totally ordered [11], we assume the labeling function $\lambda$ is not auto-concurrent; this means that two

\footnotetext{
${ }^{1}$ We will use $x<_{0} y$ as a shorthand for $x \leq_{0} y$ and $x \neq y$.
} 
concurrent events (i.e. not related by $\leq_{0}$ ) cannot have the same label ${ }^{2}$ :

$$
\forall x, y \in O, \lambda(x)=\lambda(y) \Leftrightarrow x \leq_{O} y \text { or } y \leq_{O} x
$$

Definition 1 A labeled computation $C$ is thus defined as the labeled partial order (lpo for short):

$$
C=<O, \leq_{0}, \Sigma, \lambda>
$$

When necessary, we will point out the observable events by a couple of integers: the process number on which it occurs, and its rank on this process. Figure 1 displays a distributed computation in the classical space-time diagram. Observable events are denoted by black dots; exchanges of messages are represented by arrows going from one process line to another one. Lpos

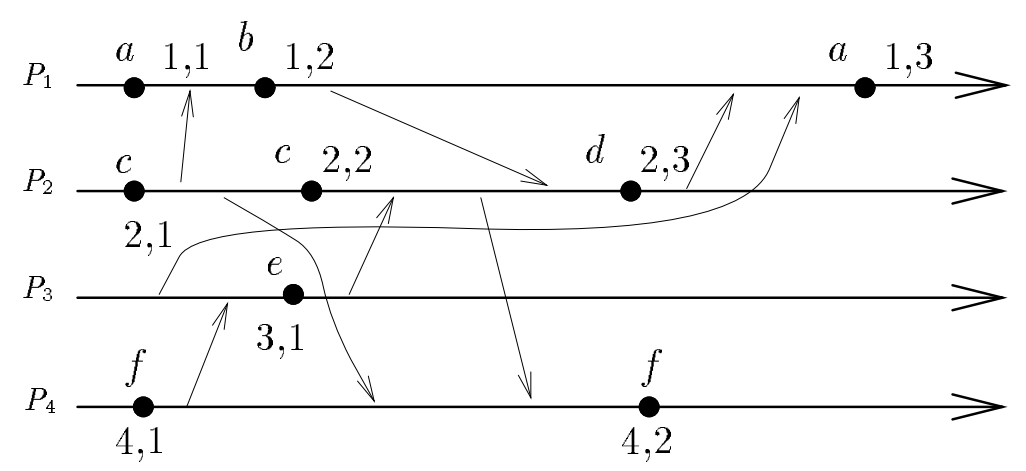

Figure 1: A distributed computation $C$.

can be usually represented by a covering graph (or Hasse diagram) ${ }^{3}$ oriented bottom-up (canonical representation). In such a graph $x<_{0} y$ iff there exists a path from $x$ to $y$. Figure 2 displays the lpo $C$ associated with the distributed computation of Figure 1.

\footnotetext{
${ }^{2}$ This is easily achieved in this model by associating with each observable event, the process number on which it occured.

${ }^{3}$ In a covering graph only non reflexive and non transitive edges are drawn, i.e. there is an edge between $x$ and $y$ iff $x<_{0} y$ and $\nexists z \in O: x<_{0} z$ and $z<_{0} y$.
}

$\mathrm{RR} \mathrm{n}^{\circ} 2595$ 
E. Fromentin, Cl. Jard, G.V. Jourdan, M. Raynal

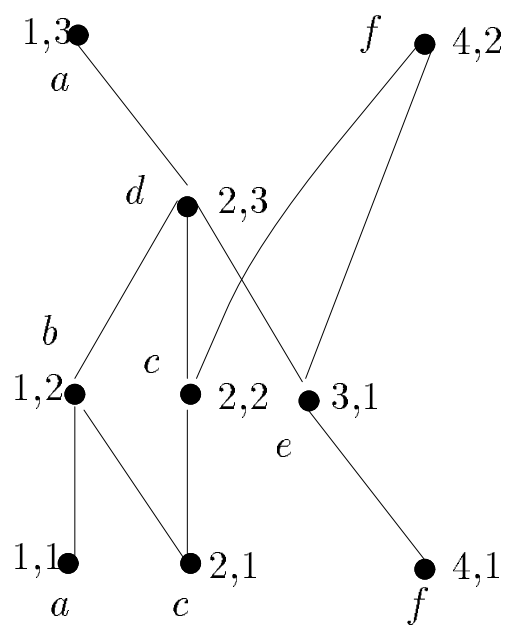

Figure 2: The covering graph of the lpo associated with $C$.

\section{Specification of properties}

\subsection{Language associated with observable events}

With each observable event $x$ can be associated the set of its causal predecessors; this set is denoted $\downarrow_{0} x$. It is formally defined as:

$$
\downarrow_{O} x=\left\{y \in O: y<_{0} x\right\}
$$

Some of the causal predecessors of $x$ constitute the set of its immediate predecessors (the predecessor nodes in the covering graph); this set is denoted by:

$$
\downarrow_{O}^{i m} x=\max _{\leq_{O}}\left(\downarrow_{0} x\right)
$$

where $y \in \max _{\leq_{O}}(X)$ iff $y \in X$ and $\nexists z \in X, y<_{O} z$ (such an $y$ is called a maximal event of $X$ ). For example, in Figure 1, the second observable event on process $P_{4}$ (event $(4,2)$ ), labeled $f$ has two immediate predecessors, one on $P_{2}$ (event $(2,2)$, labeled by $c$ ), and one on $P 3$ (event $(3,1)$, labeled by $e$ ). Note that event $(4,1)$ precedes the event $(4,2)$ but not immediately. 
More generally, the set of all maximal paths (in the covering graph) ending at an obervable event $x$ can be associated with it. These paths form words on $\Sigma^{\star}$ when considering the labeling function $\lambda$; let us denote by $\mathcal{L}(x)$ this set of words.

\section{Definition 2}

$\mathcal{L}(x)$ constitutes the language associated with the observable event $x$.

In Figure 2, we have $\mathcal{L}((1,3))=\{a b d a, c b d a, c c d a, f e d a\}$. The elements of $\mathcal{L}(x)$ correspond to the "longest control flows" that are needed to produce $x$. We can see that, in the previous example (Figure 1), the path $a b a$, including only events produced by $P_{1}$ is not a "longest control flow". The set of all these maximal sequences is sufficient to include all the observable events that causally occured before a given observable event $x$.

Additionally, considering maximal sequences provides a general framework allowing one to decide wether or not two distributed computations are different, as far as causal relationships are concerned, with respect to some property. For example, consider the two distributed executions depicted in Figure 3. In this figure, suppose that we want to check whether there is a sequence like $a b b$. We can see that, by not considering maximal paths, we are not able to distinguish between $E_{1}$ and $E_{2}$ as in both cases the answer is "yes". Whereas, by considering maximal paths, the answer is false for $E_{1}$.

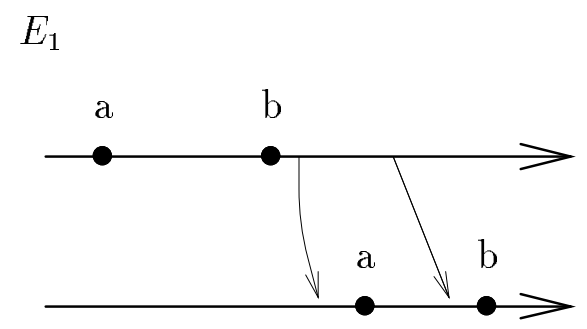

$E_{2}$

Figure 3: Distinguishing two distributed computations

RR $n^{\circ} 2595$ 


\section{2 $\quad$ Specifying properties}

Specifying properties by set of words is commonplace. We consider here regular languages, i.e. properties which can be specified by finite automata ${ }^{4}$. So a property is described by an automaton ${ }^{5} \Phi=\left(\Sigma, Q, q_{0}, \delta, F\right)$ where:

- $\Sigma$ is the alphabet (labels of observable events);

- $Q$ is the set of states;

- $q_{0}$ is the initial state;

- $\delta \subseteq Q \times A \times Q$ is the transition function;

- $F \subseteq Q$ is the set of final states.

$\mathcal{L}(\Phi)$ will denote the language recognized by $\Phi$.

Considering an observable event $x$ the basic question we want to answer concerns the validity of a maximal sequence terminating at $x$ : "Does a word of $\mathcal{L}(x)$ belong to $\mathcal{L}(\Phi)$ ?" More formally, the satisfaction rule is defined by:

\section{Definition 3 (Satisfaction rule)}

$$
\forall x \in O, x \models \Phi \Leftrightarrow \mathcal{L}(x) \cap \mathcal{L}(\Phi) \neq \emptyset
$$

Among the properties that can described by such automata we find the analogs (obtained by replacing predicates by labels) of the "linked predicates" introduced in $[10]$ and of the "atomic sequences of local predicates" introduced in [6]; (see Figure 4).

\footnotetext{
${ }^{4}$ Other kinds of specification languages could be considered. The important thing is that the concept of state can be defined (given by an operational semantics of the specification language for example). One can think to use "real" formal description techniques like SDL, State-charts, and so on.

${ }^{5}$ Note that it can be non deterministic.
} 

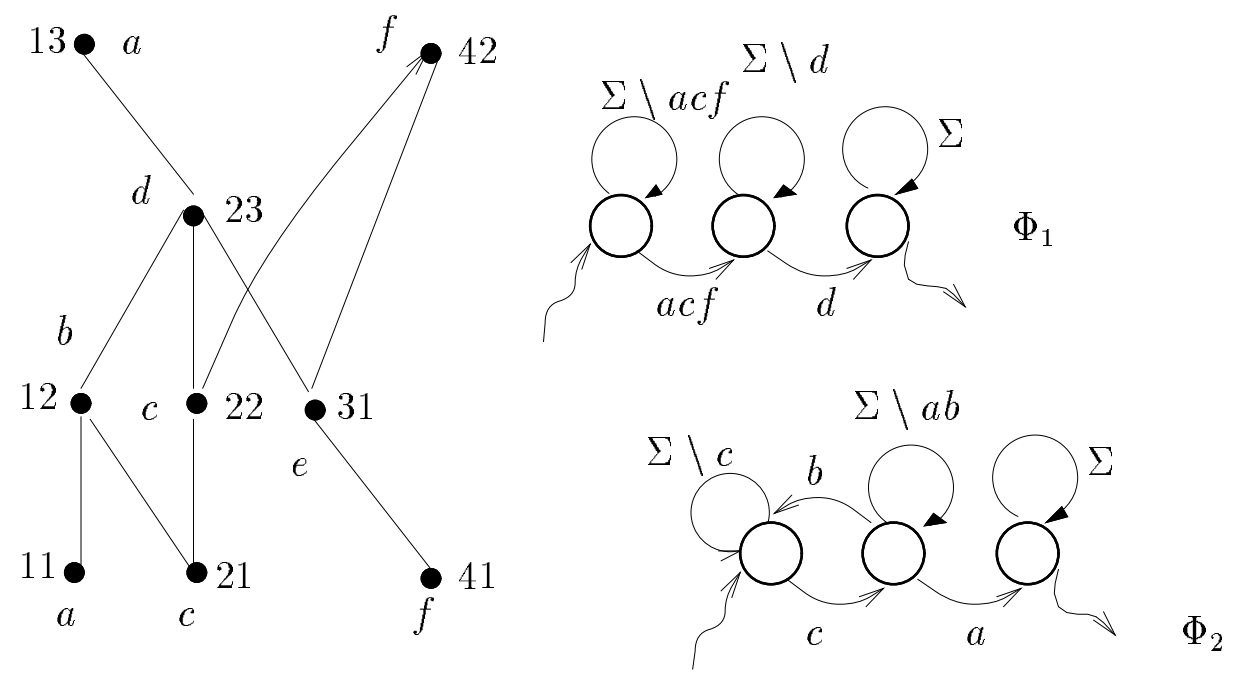

Figure 4: Detecting " $a, c$ or $f$ before $d$ " $\left(\Phi_{1}\right)$ and " $c$ before $a$ without $b$ in between" $\left(\Phi_{2}\right):(1,3) \models \Phi_{1}, \neg\left((1,3) \models \neg \Phi_{2}\right)$ and $(1,3) \models \Phi_{2}$

\section{An on-the-fly analysis algorithm}

To answer the question " $x \models \Phi$ " on the fly and without delay we have to confront a partial order, incrementally defined, to an automaton. We first show that this question can be answered by associating a set of states of the automaton with each observable event. Then we show that, for each observable event $x$, such an association can be done on-the-fly and without delay if we know the states of the automaton associated with the observable events preceding immediately $x$ (the set of these events is $\downarrow_{0}^{i m} x$ ). Consequently, the algorithm proceeds inductively along causal paths; its most subtle part resides in detecting whether an observable event is an immediate predecessor of another observable event.

RR $n^{\circ} 2595$ 


\subsection{An incremental analysis}

Consider an observable event $x$. Let $\Phi(x)$ be the set of states reached by the automaton by analyzing all words of $\mathcal{L}(x)$ :

$$
\Phi(x)=\delta^{\star}\left(q_{o}, \mathcal{L}(x)\right)
$$

where $\delta^{\star}$ is the transition function of $\Phi$ extended to words:

$$
\begin{aligned}
\delta^{\star}\left(q_{o}, u . x\right) & =\delta\left(\delta^{\star}\left(q_{o}, u\right), x\right) \\
\delta^{\star}\left(q_{o}, \epsilon\right) & =\left\{q_{o}\right\} \text { where } \epsilon \text { is the empty word } .
\end{aligned}
$$

We have:

$$
x=\Phi \Leftrightarrow \Phi(x) \cap F \neq \emptyset
$$

The important point is that $\mathcal{L}(x)$, and thus $\Phi(x)$, can be incrementally computed, as shown by the following proposition.

\section{Proposition 1}

$$
\begin{aligned}
\forall x \in O, \Phi(x) & =\delta\left(\Phi\left(\downarrow_{O}^{i m} x\right), \lambda(x)\right) \\
\Phi(\emptyset) & =\left\{q_{0}\right\}
\end{aligned}
$$

\section{Proof:}

$\mathcal{L}(x)=\mathcal{L}\left(\downarrow_{0}^{i m} x\right) \cdot \lambda(x)$ if we note $\mathcal{L}(\emptyset)=\epsilon$. Thus $\Phi(\emptyset)=\delta^{\star}\left(q_{o}, \mathcal{L}(\emptyset)\right)=\delta^{\star}\left(q_{o}, \epsilon\right)$ $=\left\{q_{o}\right\}$ by definition of $\delta^{\star}$. Second, $\Phi(x)=\delta^{\star}\left(q_{o}, \mathcal{L}\left(\downarrow_{0}^{i m} x\right) \cdot \lambda(x)\right)$, and by definition of $\delta^{\star}, \Phi(x)=\delta\left(\delta^{\star}\left(q_{o}, \mathcal{L}\left(\downarrow_{o}^{i m} x\right)\right), \lambda(x)\right)$. This leads to the proposition. 


\subsection{Principle of the algorithm}

The computation of $\Phi(x)$ for each observable event $x$ is distributed among the processes. The non local information is acquired by piggybacking control information on messages. The automaton $\Phi$ is known by all the processes. Each process $P_{i}$ is endowed with two arrays: $L O_{i}[1 . . n]$ and $S L O_{i}[1 . . n]$. The idea is that at every moment $L O_{i}[j]$ contains the last observable event on $P_{j}$ in the causal past of the current event $t \in E_{i}$. SLO $O_{i}[j]$ contains the state information $\Phi\left(L O_{i}[j]\right)$ if $L O_{i}[j]$ is a maximal event in the set of the last observable events $\bigcup_{k \in 1 . . n} L O_{i}[k] . S L O_{i}[j]$ takes the value $\emptyset$ otherwise. More precisely:

Definition $4 \forall t \in E_{i}, \forall j \in 1 . . n$

$$
\begin{aligned}
L O_{i}[j](t)= & \max _{\leq_{O}}\left(\downarrow_{E} t \cap O_{j}\right) \\
S L O_{i}[j](t)= & \Phi\left(L O_{i}[j](t)\right) \\
& \text { if } L O_{i}[j](t) \in \max _{\leq_{O}}\left(\bigcup_{k \in 1 . . n} L O_{i}[k](t)\right) \\
= & \emptyset \text { otherwise }
\end{aligned}
$$

For a process $P_{i}$, and for the next observable event $x$ that will occur on $P_{i}, L O_{i}$ gives the $n$ potential candidates to be immediate predecessors of $x$. When $x$ occurs, the information about states of the automaton is present in $S L O_{i}$. According to the previous definition, $\Phi(x)$ can be computed by using the following rule:

$$
\forall x \in O_{i}, \Phi(x)=\delta\left(\bigcup_{j \in 1 . . n} S L O_{i}[j](x), \lambda(x)\right)
$$

\subsection{Construction of the algorithm}

The algorithm may be designed inductively. Supposing that $\Phi(y)$ is correctly computed for all the observable events in the past of a current event $t \in E_{i}$, the question is to derive for each event $t$ occuring on $P_{i}$, the code that correctly computes $L O_{i}$ and $S L O_{i}$ according to their definitions.

Initially: there is no observable event.

$\forall j \in 1 . . n, L O_{i}[j]=\emptyset$ and $S L O_{i}[j]=\Phi(\emptyset)=\left\{q_{o}\right\}$.

$\mathrm{RR} \mathrm{n}^{\circ} 2595$ 
Upon observation of $x$ on $P_{i}: \quad x$ becomes the last observable event on $P_{i}$. The last observable event on $P_{j}(j \neq i)$ known by $P_{i}$, remains unchanged. Thus $L O_{i}[i]$ becomes $\{x\} . x$ is also the only maximal element of $\bigcup_{j \in 1 . . n} L O_{i}[j]$ since, by definition of $L O_{i}$, all the $L O_{i}[j]$ are in the past of $x$. Thus $S L O_{i}[i]$ becomes $\delta\left(\bigcup_{j \in 1 . . n} S L O_{i}[j](x), \lambda(x)\right)$. The other components of $L O_{i}$ are not maximal and then, by definition, $\forall j \neq i, S L O_{i}[j]$ becomes $\emptyset$.

Upon sending a message from $P_{i}: L O_{i}$ and $S L O_{i}$ do not change. They are piggybacked on the message in order to propagate control information.

Upon receiving a message from $P_{k}$, transporting $L O_{k}$ and $S L O_{k}$ : this is the most subtle part of the algorithm. We proceed componentwise by comparing the relative sequencing of the event $L O_{i}[j]$ (the last observable event on $P_{j}$, known by $P_{i}$ before reception) with respect to the event $L O_{k}[j]$ (the last observable event on $P_{j}$ known by $P_{k}$ when it sent the message).

Note that $L O_{i}[j]$ and $L O_{k}[j]$ are necessarily ordered since they occured on the same process $P_{j}$. Thus we have to consider three cases: $L O_{i}[j]<_{j} L O_{k}[j]$, $L O_{k}[j]<{ }_{j} L O_{i}[j]$ and $L O_{k}[j]=L O_{i}[j]$ (same event). These cases are illustrated in Figure 5.

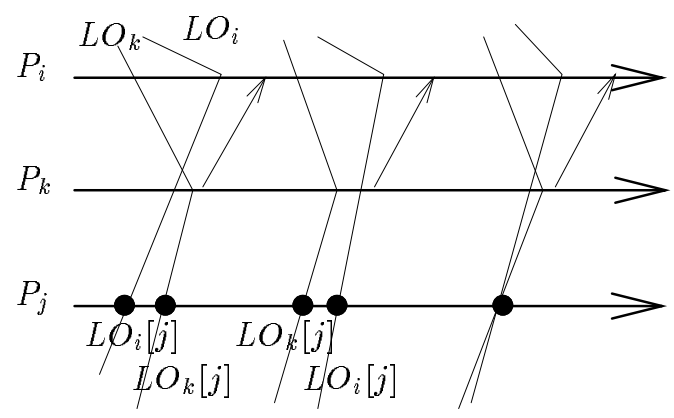

Figure 5: Relative sequencing of $L O_{i}[j]$ and $L O_{k}[j]$. 


\begin{tabular}{|l|l|l|l|l|}
\hline$S L O_{i}[j]$ & $\emptyset$ & $\times$ & $\emptyset$ & $\times$ \\
\hline$S L O_{k}[j]$ & $\emptyset$ & $\times$ & $\times$ & $\emptyset$ \\
\hline $\begin{array}{l}\text { new } \\
S L O_{i}[j]\end{array}$ & $\emptyset$ & $\times$ & $\emptyset$ & $\emptyset$ \\
\hline
\end{tabular}

Figure 6: Values of $S L O_{i}[j]$ and $S L O_{k}[j]$, and expected result.

case 1: $L O_{i}[j]<{ }_{j} L O_{k}[j]$.

In that case the last observable event on $P_{j}$ known by $P_{k}$ occured after the one known by $P_{i}$. The last observable event on $P_{j}$ known by $P_{i}$ is now $L O_{k}[j]$, and the corresponding value of $S L O_{k}[j]$ has to be registered. $L O_{i}[j]$ is updated to $L O_{k}[j]$ and $S L O_{i}[j]$ is updated to $S L O_{k}[j]$.

case 2: $L O_{k}[j]<{ }_{j} L O_{i}[j]$.

This is the symmetrical situation. The values known by $P_{i}$ must be kept. There is nothing to do.

case 3: $L O_{k}[j]=L O_{i}[j]$.

Here, $P_{i}$ and $P_{k}$ refer to the same event on $P_{j}$. Let us remark that if $S L O_{i}[j]$ and $S L O_{k}[j]$ are not empty, they have the same value: this is because the function $\Phi$ has been computed for this event by process $P_{j}$ and remained unchanged until it is set to empty. The situation is summarized in Figure 6, where we consider the respective values of $S L O_{i}[j]$ and $S L O_{k}[j]$, which are $\emptyset$ or a non empty set $X$. The event $L O_{i}[j]$ is not maximal if it is already known as non maximal by $P_{i}$ or $P_{k}$. This is why the new value of $S L O_{i}[j]$ is not empty if and only if its previous value on $P_{i}$ and $S L O_{k}[j]$ are both non empty. In that case, the value of $S L O_{i}[j]$ remains the same. In other words (see Figure 6 ), $S L O_{i}[j]$ is updated by setting it to empty only when $S L O_{i}[j] \neq \emptyset$ and $S L O_{k}[j]=\emptyset$.

$\operatorname{RR} \mathrm{n}^{\circ} 2595$ 
In the algorithm an observable event is represented by its rank on the process which produced it. In others words each $L O_{i}[j]$ can be coded by an integer. Moreover the local sequencing $<_{i}$ is then just the usual order on integers ${ }^{6}$. The algorithm is defined by the four following statements $\mathrm{S} 1$ to $\mathrm{S} 4$ executed by each process $P_{i}$.

${ }^{6}$ The array $L O_{i}$ is implemented by using the vector clocks proposed by Fidge and Mattern $[2,9]$. 


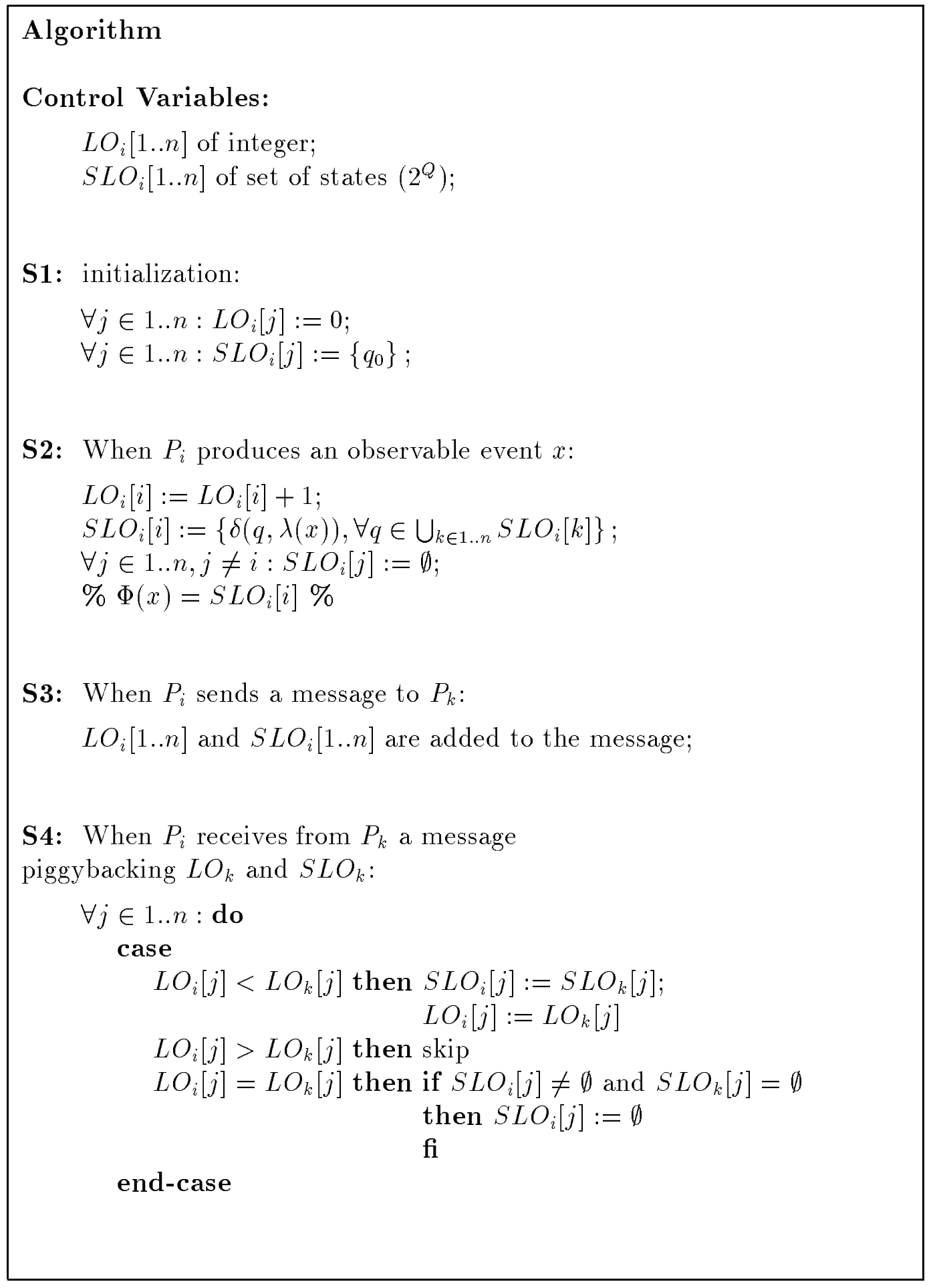

RR $n^{\circ} 2595$ 


\section{Particular Cases}

According to the position of observable events with respect to communication events, several particular cases can be defined. In all these cases the analysis algorithm simplifies.

\subsection{Non invisible process participation}

In this case we assume there is at least one observable event during any interval of a process beginning with a receive event and ending with a send event. This assumption, called "non invisible process participation", is described in Figure 7.

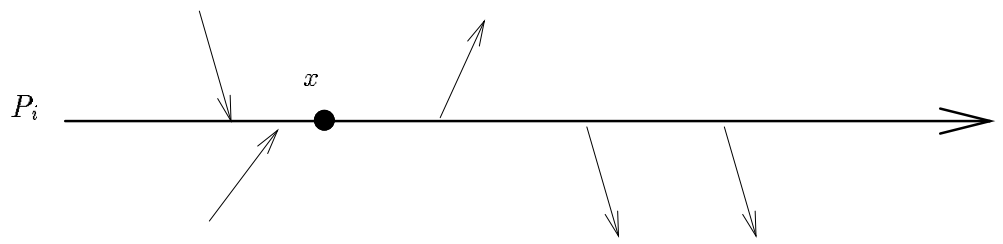

Figure 7: Non invisible process participation.

This assumption has the following immediate consequence: when a process $P_{i}$ sends a message we have always:

$$
S L O_{i}[k]:=\emptyset, \quad \forall k \in 1 . . n, \quad k \neq i
$$

It follows that only the vector $L O_{i}[1 . . n]$ and the set $S L O_{i}[i]$ have to be piggybacked. Statement S3 and $\mathrm{S} 4$ can be simplified accordingly.

\subsection{Non invisible communication}

Here we assume all communication events are observed in the following way: there is always an observable event just before every send event and just after every receive event. In that case each observable event $x$ occuring on $P_{i}$ has 
only one or two immediate predecessors: in the case of two predecessors, one on $P_{i}$ and another on the process which sent the last received message. In Figure $8, x$ has two immediate predecessors $z$ and $y ; x^{\prime}$ as only $x$ as immediate predecessor $\left(\left(t, x^{\prime}\right)\right.$ is not an edge on a "longest control flow").

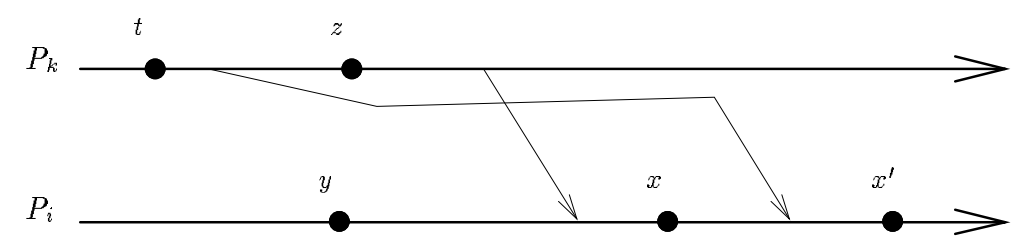

Figure 8: Non invisible communication.

It follows from this simplifying assumption that the array of sets $S L O_{i}[1 . . n]$ can be just represented by a single set, since there is only one component which is not empty.

\section{Concluding remarks}

A general algorithm working on the fly and without delay has been introduced to analyze distributed computations. It associates with each observable event $x$ of the computation the set of the longest control flows (sequences of observable events) that terminate at this event. A labeling function allows the user to consider these sequences as words on some alphabet and the algorithm checks whether these words belongs to some language (defined by a finite state automaton). It has been shown that according to the constraints on the position of observable events with respect to communication events, the analysis algorithm can be simplified.

This work is in continuation of previous works on on-the-fly distributed detection of predicates. It generalizes some of them and provides a good description power for the specification of properties while keeping an efficient and simple distributed detection algorithm. For example, the algorithms introduced in $[4,10]$ to detect linked predicates and the algorithm which detects regular

$\operatorname{RR} \mathrm{n}^{\circ} 2595$ 
patterns in distributed computations introduced in [3] are two particular cases which consider all control flows ending at observable events (and not only the maximal ones). Moreover, it appears that the algorithm described in [1] that computes the immediate predecessors of an observable event $x$ constitutes the core of the verification algorithm that has been introduced.

\section{Acknowledgements}

We are grateful to S. Lorcy and N. Plouzeau for interesting discussions related to this paper. This work has been supported in part by the Commission of European Communities under ESPRIT Programme BRA 6360 (BROADCAST), by the French CNRS under the grant Parallel Traces and by a French-Israeli grant on distributed computing.

\section{References}

[1] C. Diehl, C. Jard, and J. X. Rampon. Reachability analysis on distributed executions. In Theory and Practice of Software Development, pages 629643, TAPSOFT, Springer Verlag, LNCS 668 (Gaudel and Jouannaud editors), April 1993.

[2] J. Fidge. Timestamps in message passing systems that preserve the partial ordering. In Proc. 11th Australian Computer Science Conference, pages 55-66, February 1988.

[3] E. Fromentin, M. Raynal, V.K. Garg, and A.I. Tomlinson. On the fly testing of regular patterns in distributed computations. In Proc. of the $23^{\text {rd }}$ International Conference on Parallel Processing, pages 73-76, St. Charles, IL, August 1994.

[4] V. K. Garg and B. Waldecker. Detection of unstable predicates in distributed programs. In Twelfth International Conference on Foundations of Software Technology and Theoretical Computer Science, pages 253-264, Springer Verlag, LNCS 625, New Delhi, India, December 1992. 
[5] M. Hurfin, N. Plouzeau, and M. Raynal. A debugging tool for distributed Estelle programs. Journal of Computer Communications, 16(5):328-333, May 1993.

[6] M. Hurfin, N. Plouzeau, and M. Raynal. Detecting atomic sequences of predicates in distributed computations. In Proc. ACM/ONR Workshop on Parallel and Distributed Debugging, pages 32-42, San Diego, CA, May 1993. (Reprinted in SIGPLAN Notices, Dec. 1993).

[7] C. Jard, T. Jeron, G.V. Jourdan, and J.X. Rampon. A general approach to trace-checking in distributed computing systems. In Proc. 14th IEEE Int. Conf. on DCS, pages 396-403, Poznan, Poland, June 1994.

[8] L. Lamport. Time, clocks and the ordering of events in a distributed system. Communications of the ACM, 21(7):558-565, July 1978.

[9] F. Mattern. Virtual time and global states of distributed systems. In Cosnard, Quinton, Raynal, and Robert, editors, Parallel and Distributed Algorithms, pages 215-226, North-Holland, October 1988.

[10] B.P. Miller and J. Choi. Breakpoints and halting in distributed programs. In Proc. $8^{\text {th }}$ IEEE Int. Conf. on Distributed Computing Systems, San Jose, pages 316-323, July 1988.

[11] V. Pratt. Modelling concurrency with partial orders. Int. Journal of Parallel Programming, 15(1), November 1986.

$\operatorname{RR} \mathrm{n}^{\circ} 2595$ 


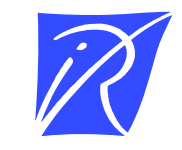

Unité de recherche INRIA Lorraine, Technopôle de Nancy-Brabois, Campus scientifique, 615 rue du Jardin Botanique, BP 101, 54600 VILLERS LES NANCY

Unité de recherche INRIA Rennes, Irisa, Campus universitaire de Beaulieu, 35042 RENNES Cedex

Unité de recherche INRIA Rhône-Alpes, 46 avenue Félix Viallet, 38031 GRENOBLE Cedex 1

Unité de recherche INRIA Rocquencourt, Domaine de Voluceau, Rocquencourt, BP 105, 78153 LE CHESNAY Cedex

Unité de recherche INRIA Sophia-Antipolis, 2004 route des Lucioles, BP 93, 06902 SOPHIA-ANTIPOLIS Cedex

Éditeur

INRIA, Domaine de Voluceau, Rocquencourt, BP 105, 78153 LE CHESNAY Cedex (France)

ISSN 0249-6399 